\title{
Design and Analysis Strategies for Digital Repetitive Control Systems with Time-Varying Reference/Disturbance Period
}

\author{
Ramon Costa-Castelló, Josep M. Olm and Gemán A. Ramos
}

\begin{abstract}
This article introduces and analyzes the performance features of different design schemes for digital repetitive control systems subject to references/disturbances that exhibit non-uniform frequency. Aiming for the maintenance of a constant value for the ratio $T_{p} / T_{s}$, where $T_{p}$ is the period of the reference/disturbance signal and $T_{S}$ is the sampling period, two approaches are proposed. The first one deals with the realtime adaptation of $T_{S}$ to the actual changes of $T_{p}$; the stability issue is studied by means of an LMI gridding method and also using robust control techniques. The second one propounds the introduction of an additional compensator that annihilates the effect of the time-varying sampling in the closed-loop system and forces its behavior to coincide with the one corresponding to an a priori selected nominal sampling period; the procedure needs the internal stability of the compensator-plant subsystem, which is checked by means of LMI gridding. The theoretical results are experimentally tested and compared through a mechatronic plant model.
\end{abstract}

\section{INTRODUCTION}

Repetitive control [1] is an Internal Model Principle-based control technique that allows both the tracking and rejection of periodic signals. Essentially, this is achieved including a generator of the reference/disturbance in the control loop.

It is usual to design repetitive controllers assuming a fixed period $T_{p}$ for the signals to be tracked/rejected. Then, a fixed sampling period $T_{S}$ is selected and, eventually, the value of the ratio $N=T_{p} / T_{s}$ is structurally embedded in the control algorithm. However, it is well known that even slight changes in the frequency of the tracked/rejected signals result in a dramatic decay of the controller performance.

The approaches that have dealt with this problem may be grouped in two main frameworks, namely, the ones that propound to maintain $T_{S}$ constant and adapt the value of $N$ according to the time variation of $T_{p}$ [2], [3], and the ones that work with a fixed value for $N$. The main advantage of setting a constant value for $N$ is quality preservation in signal reconstruction, and this has lead the authors to select such an option throughout this article.

Existing literature dealing with the maintenance of a constant $N$ considers, in turn, two different paths. The first proposal keeps the initially selected sampling time $T_{s}$, and

R. Costa-Castelló is the Institute of Industrial and Control Engineering, Universitat Politècnica de Catalunya, Av. Diagonal, 64708028 Barcelona, Spain ramon. costa@upc.edu

J. M. Olm, corresponding author, is with the Department of Applied Mathematics IV, Universitat Politècnica de Catalunya, Avda. Víctor Balaguer, s/n, 08800 Vilanova i la Geltrú, Spain josep.olm@upc.edu

G.A. Ramos is with the Department of Electrical and Electronic Engineering, Universidad Nacional de Colombia, Bogotá DC, Colombia garamosfeunal. edu. co

This work is partially supported by the spanish Ministerio de Educación y Ciencia (MEC) under project DPI2007-62582 robustness is achieved by means of large memory elements [4] or introducing a fictitious sampler operating at a variable sampling rate and later using a fixed frequency internal model [5]. The second idea proposes to adapt the controller sampling rate according to the reference/disturbance period [6], [3]. This allows to preserve the steady-state performance while maintaining a low computational cost but, on the other hand, it implies structural changes in the system behavior that may destabilize the closed-loop system. Indeed, no formal stability proofs are provided in the existing literature for this case.

In order to cope with the problems inherent to the preservation of a constant value for $N$, two different schemes are proposed and comparatively studied in this article. The first approach consists of adapting the value of $T_{S}$ according to the variation of $T_{p}$, which is detected by means of a frequency observer, and perform an accurate analysis of stability margins where reliable performance is definitely ensured. The stability analysis is carried out twofold, namely, using a Linear Matrix Inequality (LMI) gridding method and also with robust control techniques. Alternatively, this adaptation strategy may also include an additional compensator placed between the controller and the plant that, under the assumption of internal stability, annihilates the effect of the time-varying period and forces the closed-loop behavior to match that of a pre-selected nominal sampling period. The theoretical predictions are validated and comparatively analyzed through a mechatronic plant model [7], for which experimental results have been obtained.

\section{DIGITAL REPETITIVE CONTROL UNDER CONSTANT SAMPLING PERIOD}

Repetitive controllers are composed by two main elements: the internal model, $G_{1}(z)$, and the stabilizing controller, $G_{2}(z)$. The internal model is the one in charge of guaranteeing null or small error in steady state, while the stabilizing controller assures closed-loop stability. In this work the generic internal model will be used, i.e. $G_{1}(z)$ is selected as:

$$
G_{1}(z)=\frac{H(z)}{z^{N}-H(z)} .
$$

$H(z)$ plays the role of a low-pass filter in charge of introducing robustness in the high frequency range [8]. Although the internal model and the stabilizing controller can be arranged in different ways, most repetitive controllers are usually implemented in a "plug-in" fashion, as depicted in Figure 1: the repetitive compensator is used to augment an existing 


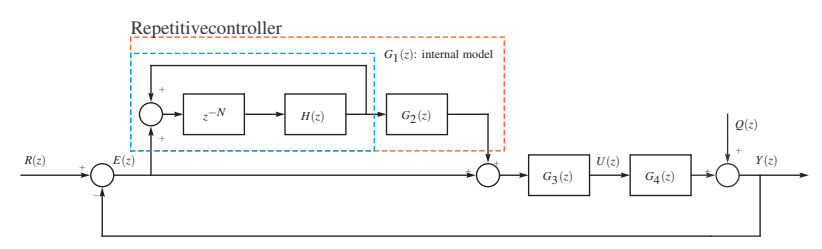

Fig. 1. Discrete-time block-diagram of the proposed repetitive transfer function.

nominal controller, $G_{3}(z)$. This nominal compensator is designed to stabilize the plant, $G_{4}(s)$, and provides disturbance attenuation across a broad frequency spectrum.

Assume that both $T_{p}$ and $T_{s}$ are constant, which makes $N$ also constant, and let $G_{4}(z)$ stand for the corresponding z-transform of $G_{4}(s)$. Sufficient stability criteria are given in the next Proposition:

Proposition 1: ([7], [9])The closed-loop system of Figure 1 is stable if the following conditions are fulfilled:

1) The closed-loop system without the repetitive controller $G_{o}(z)$ is stable, where

$$
G_{o}(z)=\frac{G_{3}(z) G_{4}(z)}{1+G_{3}(z) G_{4}(z)}
$$

2) $\|H(z)\|_{\infty}<1$.

3) $\left\|1-G_{o}(z) G_{2}(z)\right\|_{\infty}<1$, where $G_{2}(z)$ is a design filter to be chosen.

\section{DIGITAL REPETITIVE CONTROL UNDER TIME-VARYING SAMPLING PERIOD}

The repetitive controller introduced in the previous section contains the ratio $N=T_{p} / T_{s}$, which is embedded in the controller implementation. This setting renders a well-known good performance if the reference/disturbance periodic signal has a known constant period. However, the controller performance decays dramatically when a variation of $T_{p}$ appears. This section deals with the adaptation of the controller sampling time $T_{S}$ following the reference/disturbance period $T_{p}(t)$, with the aim of maintaining a constant value for $N$. Hence, on the one hand, $G_{1}(z), G_{2}(z)$ and $G_{3}(z)$ are designed and implemented to provide closed-loop stability for a nominal sampling time $T_{s}=\bar{T}$, in accordance with Proposition 1; their structures remain always invariant, i.e. they experiment no further structural changes. On the other hand, the period of the sampler device that precedes the plant $G_{4}(s)$ is accommodated to the variation of $T_{p}(t)$; therefore, its discrete-time representation is that of a Linear Time Varying (LTV) system. The accommodation scheme is detailed in Figure 2.

However, although the proposed technique allows to adapt the system to the specific signal frequency to be tracked/rejected without changing the digital controller, the sampling-rate change may compromise closed-loop stability. In what follows, a closed-loop stability analysis of the system under varying sampling rate condition is carried out.

Let the discrete-time state-space representations of the blocks $G_{i}$ (see Figure 1 ) be denoted by $\left(A_{i}, B_{i}, C_{i}, D_{i}\right), i=$

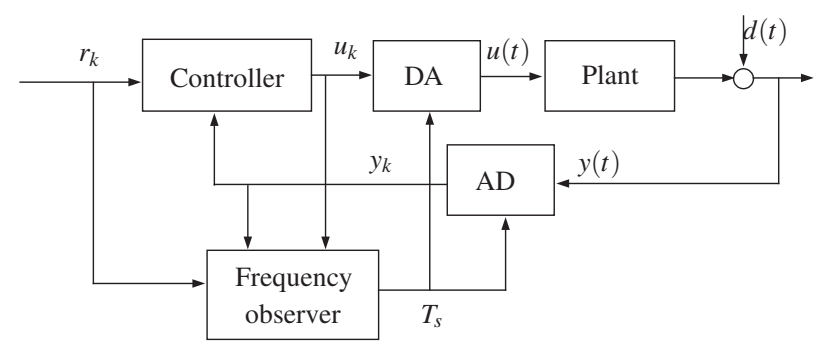

Fig. 2. Accommodation of the sampling period $T_{s}$ to possible variations of $T_{p}$.

$1,2,3,4$. The closed-loop system state equations are derived under the following assumptions:

- $D_{1}=D_{4}=0$.

- The representations corresponding to blocks $G_{1}(z)$, $G_{2}(z)$ and $G_{3}(z)$ are obtained from the nominal sampling time $T_{S}=\bar{T}$ and remain constant $\forall t$.

- Only the plant discrete-time model matrices $A_{4}, B_{4}$, vary according to sampling rate updating: $A_{4}=A_{4}\left(T_{S}\right), B_{4}=$ $B_{4}\left(T_{S}\right)$, while $C_{4}$ is maintained constant. Hence, assuming that $(A, B, C, 0)$ stands for the continuous-time plant state-space representation, i.e. $G_{4}(s)=C(s \mathbb{I}-A)^{-1} B$, then

$$
A_{4}(T) \triangleq e^{A T}, \quad B_{4}(T) \triangleq \int_{0}^{T} e^{A r} B d r .
$$

Let the system be sampled at time instants $\left\{t_{0}, t_{1}, \ldots\right\}$, with $t_{0}=0, t_{k+1}>t_{k}$, the sampling periods being $T_{k}=t_{k+1}-t_{k}$. Let also $x_{k} \triangleq x\left(t_{k}\right), r_{k} \triangleq r\left(t_{k}\right), y_{k} \triangleq y\left(t_{k}\right)$. The state equations are given by a discrete-time LTV system of the form:

$$
x_{k+1}=\Phi\left(T_{k}\right) x_{k}+\Pi\left(T_{k}\right) r_{k}, \quad y_{k}=\Upsilon x_{k} .
$$

Assume that $G_{1}(z), G_{2}(z)$ and $G_{3}(z)$ are designed according to Proposition 1 to provide stability for a nominal sampling time $T_{s}=\bar{T}$. Hence, when $T_{k}=\bar{T}, \forall k$, the overall system is stable by construction. A methodology for studying the closed-loop behavior under non-uniform sampling period is developed in this Section.

Proposition 2 ([10]): Let the sampling period, $T_{k}$, take values in a compact subset $\mathscr{T} \subset \mathbb{R}^{+}$. If there exists a matrix $P$ such that

$$
L_{T_{k}}(P)=\Phi\left(T_{k}\right)^{\top} P \Phi\left(T_{k}\right)-P<0 \text {, s.t. } \quad P=P^{\top}>0,
$$

$\forall T_{k} \in \mathscr{T}$, then (3) is internally stable and uniformly Bounded Input-Bounded Output (BIBO) stable in $\mathscr{T}$.

\section{A. LMI gridding-based stability analysis}

It is immediate to realize that relation (4) in Proposition 2 yields an infinite set of LMIs. The gridding approach introduced in [11], [12] allows a simplified stability analysis that may be performed in two stages, if necessary.

In a first stage, advantage is taken from the fact that system (3) is stable by construction for $T_{k}=\bar{T}, \forall k$. For, consider the LMI problem

$$
L_{\bar{T}}(P) \leq-\alpha \mathbb{I}, \quad \text { s.t. } \quad P=P^{\top}>0,
$$


with $L_{\bar{T}}(P)$ constructed from (4). Let $P=P_{N}$ be a feasible solution of (5) for a fixed $\alpha \in \mathbb{R}^{+}$: its existence is guaranteed in [13]. Let also $\left\{\tau_{0}, \ldots, \tau_{q}\right\}$, with $\tau_{i+1}>\tau_{i}$, be a sufficiently fine grid of $\mathscr{T}$. Then, if $L_{\tau_{i}}\left(P_{N}\right)<0, \forall i=0, \ldots, q$, stability $\forall T_{k} \in \mathscr{T}$ may be probably inferred.

Otherwise, in case that there exists at least a single $\tau_{i}$ such that $L_{\tau_{i}}\left(P_{N}\right) \geq 0$, the gridding procedure proposed in [12] may be carried out as follows. Let $\left\{\tau_{0}, \ldots, \tau_{r}\right\}$, be a sorted set of candidate sampling periods suitably distributed in $\mathscr{T}$. Then, one may solve the following finite set of LMIs:

$$
L_{\tau_{i}}(P) \leq-\alpha \mathbb{I}, \quad i=0, \ldots, r, \quad \text { s.t. } P=P^{\top}>0,
$$

for a fixed $\alpha \in \mathbb{R}^{+}$. In case that the problem is feasible and a solution, $P=P_{G}$, is encountered, the negative-definite character of $L_{T_{k}}\left(P_{G}\right)$ is to be checked for intermediate values of $T_{k}$ in each open subinterval $\left(\tau_{i}, \tau_{i+1}\right)$. If this fails to be accomplished, (6) has to be solved again for a finer grid of $\mathscr{T}$. Otherwise, the procedure should be relaunched for a new interval $\mathscr{T}^{\prime} \subset \mathscr{T}$.

\section{B. Robust control theory-based stability analysis}

The stability analysis follows the approach proposed in [14], [15], where the non-uniform sampling is viewed as a nominal sampling period affected by an additive disturbance. In the present case, the repetitive control system is designed to provide closed-loop stability for a nominal case. Then, the actual problem is to quantify the "amount" of disturbance due to aperiodical sampling that the system can accommodate while preserving stability.

Let $T=\bar{T}$ be a fixed sampling period and define $\theta_{k}=$ $T_{k}-\bar{T}$. Then, the matrix $\Phi\left(T_{k}\right)$ in (3) may be written as

$$
\Phi\left(T_{k}\right)=\Phi(\bar{T})+\tilde{\Delta}\left(\theta_{k}\right) \Psi(\bar{T}),
$$

where $\Psi(\bar{T})$ is a constant matrix and

$$
\tilde{\Delta}(\theta) \triangleq\left(\begin{array}{cc}
0 & 0 \\
0 & \Delta(\theta)
\end{array}\right), \quad \Delta(\theta) \triangleq \int_{0}^{\theta} e^{A r} d r .
$$

Notice that, using (7), (3) may be written as

$$
x_{k+1}=\left[\Phi(\bar{T})+\tilde{\Delta}\left(\theta_{k}\right) \Psi(\bar{T})\right] x_{k},
$$

which allows the following interpretation [14]: (9) can be regarded as the LTI system

$$
\Sigma:=\left\{\begin{array}{l}
x_{k+1}=\Phi(\bar{T}) x_{k}+u_{k} \\
v_{k}=\Psi(\bar{T}) x_{k},
\end{array}\right.
$$

$G_{\bar{T}}(z)=\Psi(\bar{T})[z \mathbb{I}-\Phi(\bar{T})]^{-1}$ being its associated discretetime transfer function, receiving the time-varying output feedback control action $u_{k}=\tilde{\Delta}\left(\theta_{k}\right) v_{k}$.

In what follows, $\|\cdot\|$ denotes the 2-norm of a matrix, while the $H_{\infty}$-norm of a transfer function matrix $G(z)$ is defined as $\|G(z)\|_{\infty}=\max \left\{\left\|G\left(e^{j \omega T_{s}}\right)\right\|, \forall \omega \in \mathbb{R}\right\}$.

Theorem 3: Let

$$
\gamma_{\bar{T}}=(1+\varepsilon)\left\|G_{\bar{T}}(z)\right\|_{\infty}, \quad \varepsilon>0,
$$

be an upper bound of the $H_{\infty}$-norm of system $\Sigma(10)$, and let also $\mathscr{T} \subset \mathbb{R}^{+}$be compact. If $\gamma_{\bar{T}}\|\Delta(T-\bar{T})\| \leq 1, \forall T \in \mathscr{T}$, then (3) is internally stable and uniformly BIBO stable in $\mathscr{T}$.

Proof: It follows from [14] and Proposition 2.

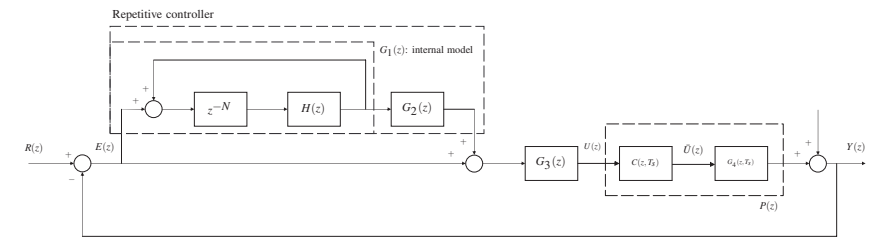

Fig. 3. Discrete-time block-diagram of the closed-loop system with the adaptation-compensation controller structure.

\section{ADAPTATION-COMPENSATION SCHEME UNDER TIME-VARYING SAMPLING}

The designed scheme takes advantage of the basic adaptive structure considered in Section III and introduces an additional compensator between the controller $G_{3}(z)$ and the plant $G_{4}(z)$, as depicted in Figure 3. The aim is to annihilate the effect of the time-varying sampling and force an output behavior corresponding to that of the nominal sampling $\bar{T}$.

Namely, let the repetitive controller be designed and implemented to provide closed-loop stability for an a-priori selected nominal sampling time $\bar{T}$ to the nominal, LTI plant

$$
G_{4}(z, \bar{T}) \triangleq \frac{\operatorname{Num}(z, \bar{T})}{\operatorname{Den}(z, \bar{T})}
$$

in accordance with Proposition 1. Moreover, assume that, in order to maintain a constant value for the ratio $N=T_{p} / T_{s}$, the controller sampling time $T_{s}$ is accommodated to the time variation of the reference/disturbance period $T_{p}(t)$, i.e. $T_{s}(t)=T_{p}(t) / N$. Therefore, the discrete-time representation of the plant $G_{4}(s)$ is that of a LTV system $^{1}$

$$
G_{4}\left(z, T_{s}\right)=\frac{\operatorname{Num}\left(z, T_{s}\right)}{\operatorname{Den}\left(z, T_{s}\right)}
$$

Finally, the structural changes caused by the time variation of $T_{s}$ are annihilated through the additional compensator

$$
C\left(z, T_{S}\right)=G_{4}(z, \bar{T}) G_{4}^{-1}\left(z, T_{S}\right)=\frac{\operatorname{Num}(z, \bar{T})}{\operatorname{Den}(z, \bar{T})} \cdot \frac{\operatorname{Den}\left(z, T_{s}\right)}{\operatorname{Num}\left(z, T_{S}\right)}
$$

that premultiplies the LTV plant $G_{4}\left(z, T_{S}\right)$. The goal of $C\left(z, T_{S}\right)$ is to compensate the variation of the plant dynamics due to aperiodical sampling. Note that in case of periodic sampling, i.e. $T_{S}=\bar{T}$, it results that $C(z, \bar{T})=1$. Although in (13) the plant inverse is used, in practice the plant dynamics is not completely cancelled. Thence, under the assumption of internal stability for the compensator-plant system

$$
P(z) \triangleq C\left(z, T_{S}\right) G_{4}\left(z, T_{S}\right)=G_{4}(z, \bar{T}),
$$

its behavior is that of the nominal LTI system $G_{4}(z, \bar{T})$. Figure 4 depicts the compensator-plant subsystem.

Proposition 4: Let $G_{4}(s)$ be a continuous LTI plant and let $G_{4}\left(z, T_{s}\right)$ be its discrete-time transfer function corresponding

\footnotetext{
${ }^{1}$ The use of the z-transform notation in a LTV framework is not formally correct. This description is here preserved in order to achieve a compact and simple notation. Hence, $z^{-1}$ should be read as a one sample time delay, but the sampling interval may change from sample to sample.
} 


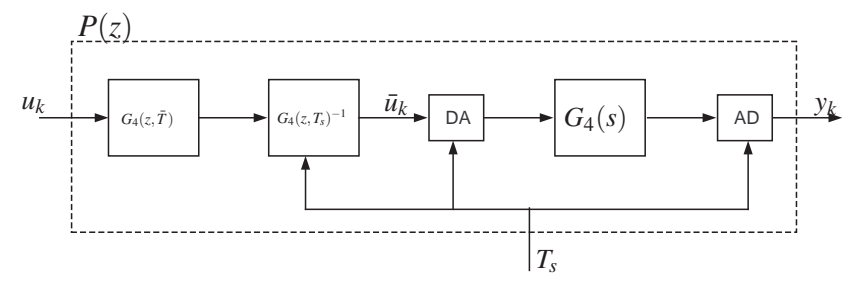

Fig. 4. Detail of the compensator-plant system.

to the sampling time $T_{s}$. Let also $T_{s}=\bar{T}$ be a nominal sampling time, and let $G_{4}(z, \bar{T})$ defined in (12) be its associated discrete-time transfer function. Assume that the compensator $C\left(z, T_{S}\right)$ of Figure 3 is defined as in (14), and that the subsystem $P(z)$ defined in (15) is internally stable for all $T_{s} \in \mathscr{T} \subset \mathbb{R}^{+}$. Finally, assume that the repetitive controller elements $G_{1}(z), G_{2}(z), G_{3}(z)$ are designed according to Proposition 1 in order to provide closed loop stability to the LTI system $P(z)$. Then, the closed-loop system depicted in Figure 3 is stable for all $T_{s} \in \mathscr{T}$.

Remark 1: (i) The internal stability of the compensatorplant subsystem $P(z)$ can be checked using the LMI gridding approach introduced in Subsection III-A.

(ii) In most practical applications the plant $G_{4}(z)$ admits a relative degree 1 , first order model description, which ensures internal stability for $P(z)$ whenever $G_{4}(z)$ is stable.

\section{EXPERIMENTAL SETUP AND RESULTS}

\section{A. Plant description}

Systems with rotary elements are usually affected by periodic disturbances due the movement of these parts. This kind of system is supposed to be moving, in some cases, at a fixed angular speed. Under these working conditions any friction, unbalance or asymmetry appearing on the system generates a periodic disturbance that affects its dynamical behavior. Reference [7] contains a description of a mechatronic plant designed to reproduce this working conditions. This device is composed of a bar holding a permanent magnet in each end, with each magnet magnetically oriented in the opposite way, and attached to a DC motor and two fixed permanent magnets. The rotation of the DC motor causes a pulsating load torque $\left(\Gamma_{p}\right)$ that depends on the mechanical angle $\theta$ of the motor axis. When the motor axis angular speed $\omega$ is constant $(\ddot{\theta}=\dot{\omega}=0)$, the pulsating torque is a periodic signal with a fundamental period directly related to the axis speed: $T_{p}=\omega^{-1}$, with $\omega$ expressed in rev/s. The control goal for this plant is maintaining the motor axis angular speed constant at a desired value.

\section{B. Control design}

A first-order model for the plant is experimentally derived from its open-loop time response without the fixed magnets:

$$
G_{4}(s)=\frac{K}{\tau s+1}=\frac{8.762}{0.10667 s+1} \frac{\mathrm{rev} / \mathrm{s}}{\mathrm{V}}
$$

The controller is constructed from (16), for a speed of $\omega=8 \mathrm{rev} / \mathrm{s}$ and obtaining 25 samples per period, i.e. $N=$

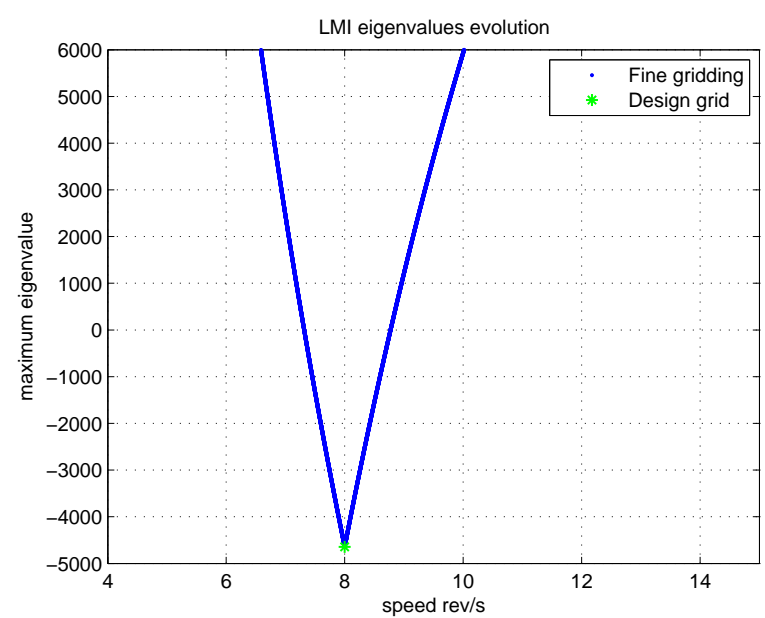

Fig. 5. Maximum eigenvalue of $L_{T_{k}}\left(P_{N}\right)$ with $\alpha=4645$ and $\bar{T}=0.005 \mathrm{~s}$.

25. These conditions imply a nominal sampling period of $\bar{T}=T_{p} / N=(\omega N)^{-1}=5 \mathrm{~ms}$. Under these assumptions the nominal discrete time plant is:

$$
G_{4}(z)=G_{4}(z, \bar{T})=\frac{K\left(1-e^{-\bar{T} / \tau}\right)}{z-e^{-\bar{T} / \tau}}=\frac{0.4012}{z-0.9542} .
$$

According to [7], [9], the following design issues have been taken into account:

- $G_{3}(z)=0.25$ provides a very robust inner loop.

- The first order null-phase FIR filter $[H(z)=0.02 z+$ $0.96+0.02 z^{-1}$ provides good performance in the present case.

- The fact that $G_{4}(z)$ is minimum-phase allows $G_{2}(z)=$ $k_{r} G_{o}^{-1}(z)$, with $k_{r}=0.3$.

These settings yield the control law:

$$
\begin{aligned}
u_{k}= & 0.25 e_{k}+0.015 e_{k-23}+0.70 e_{k-24}+ \\
& -0.84 e_{k-25}-0.018 e_{k-26}+0.02 u_{k-24}+ \\
& +0.96 u_{k-25}+0.02 u_{k-26}
\end{aligned}
$$

with $e_{k}=r_{k}-y_{k}$, where $y_{k}$ is the system output (speed) and $r_{k}$ is the reference.

\section{Adaptation of the sampling period: stability analysis}

1) LMI gridding approach: Although the controller is designed to regulate the speed at $8 \mathrm{rev} / \mathrm{s}$, in practice it will be necessary to move from this design point. Let us assume that we are interested in varying the speed reference in the interval $[6,13] \mathrm{rev} / \mathrm{s}$ : this entails a sampling period variation in the interval $\mathscr{T}=[3.63,7.87] \mathrm{ms}$.

The stability analysis that stems from Subsection IIIA includes the solution of the LMI (5), which is known to be feasible, and the checking of the negative-definite character of $L_{T_{k}}\left(P_{N}\right)$. Figure 5 shows the evolution of the maximum modulus eigenvalue of $L_{T_{k}}\left(P_{N}\right)$ when solving for $\alpha=4645$, and also for 50000 uniformly distributed values of $T_{k}$. Therefore, it can be presumed that the closed-loop system may operate in a speed range of $[7.32,8.77] \mathrm{rev} / \mathrm{s}$ with dynamically preserved stability. 


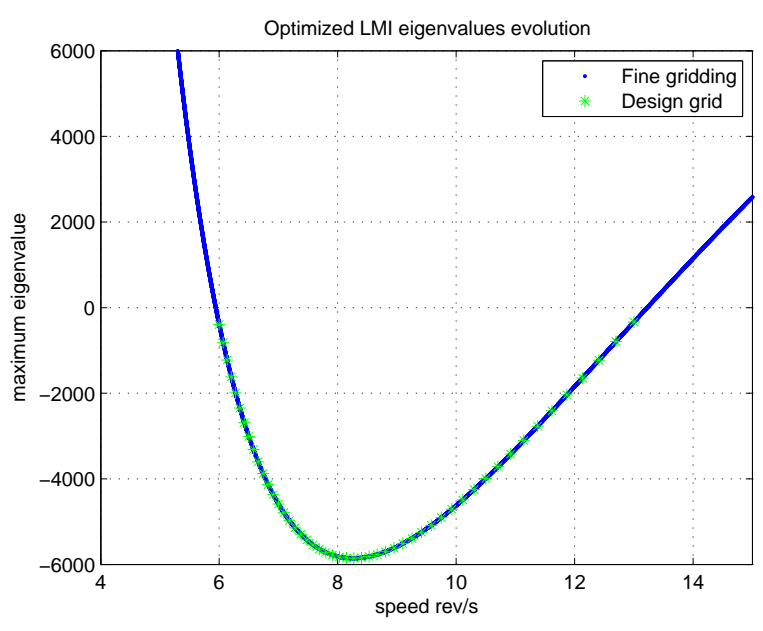

Fig. 6. Maximum eigenvalue of $L_{T_{k}}\left(P_{G}\right)$.

In order to guarantee a broader stability interval, the second method described in Subsection III-A may be applied. Therefore, 40 uniformly distributed points are selected in $\mathscr{T}=[0.00363,0.00787] \mathrm{rev} / \mathrm{s}$. These points are used to construct the set of LMIs (6), and a feasible solution $P=P_{G}$ with $\alpha=100$ is obtained. Figure 6 depicts the maximum modulus eigenvalue of $L_{T_{k}}\left(P_{G}\right)$, detailing with a star the 40 points leading to the LMI formulation. The maximum modulus eigenvalue of $L_{T_{k}}\left(P_{G}\right)$ corresponding to a finer grid consisting of 50000 uniformly distributed point are also drawn in Figure 6. These points are used to check the sign of $L_{T_{k}}\left(P_{G}\right)$ in the intervals between the points defining the LMI set. It can be seen that $L_{T_{k}}\left(P_{G}\right)<0$ for every point in this finer grid of the interval $\mathscr{T}$; hence, stability is dynamically preserved therein. This method extends the previously obtained stability interval $[7.32,8.77] \mathrm{rev} / \mathrm{s}$, thus providing less conservative results. However, this LMI approach does not provide sufficient stability conditions and may entail numerical problems. Hence, the robust control approach results are given in next item.

2) Robust control theory approach: Following Subsection III-B, the settings for the plant and the repetitive controller yield $\left\|G_{\bar{T}}(z)\right\|_{\infty}=222.1471$. In order to define $\gamma_{\bar{T}}$ (see (11)), $\varepsilon=0.0001$ has been selected. Furthermore, the plant being first order yields a scalar value for its continuous-time system matrix: $A=-9.3747$; hence, an exact bounding of $\| \Delta(T-$ $\bar{T}) \|$ is possible. The obtained numerical values are $\mathscr{T}=$ $[0.6,9.6] \mathrm{ms}$, which indicates that the performance of the device is ensured in the range $\omega \in[4.2039,59.8475] \mathrm{rev} / \mathrm{s}$.

\section{Adaptation-compensation of the sampling period}

According to Section III, the compensator $C\left(z, T_{S}\right)$ is designed as follows. On the one hand, recalling (12), the discrete-time model of the plant at the nominal sampling period $G_{4}(z, \bar{T})$ defined in (17) is taken as the time-invariant
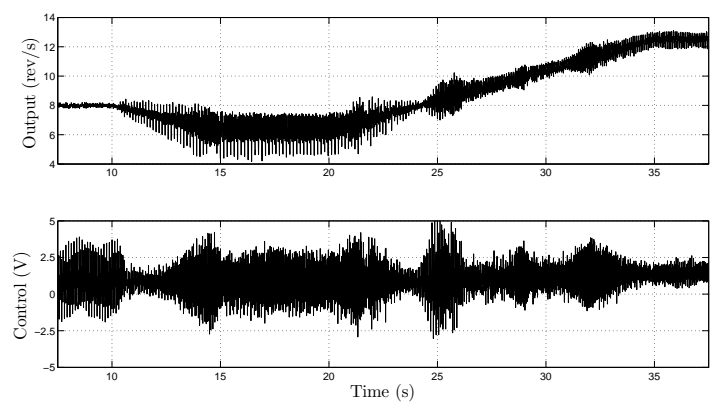

Fig. 7. Closed-loop system behavior using a repetitive controller and with sampling period fixed at the nominal value $(\bar{T}=5 \mathrm{~ms})$.

component of $C\left(z, T_{S}\right)$. On the other hand, the plant (see (13))

$$
G_{4}\left(z, T_{s}\right)=\frac{\operatorname{Num}\left(z, T_{s}\right)}{\operatorname{Den}\left(z, T_{s}\right)}=\frac{K\left(1-e^{-T_{s} / \tau}\right)}{z-e^{-T_{s} / \tau}}
$$

is first order, stable and minimum phase; hence, its inversion is possible and one can define:

$$
\frac{\operatorname{Den}\left(z, T_{S}\right)}{\operatorname{Num}\left(z, T_{s}\right)}=\frac{z-e^{-T_{S} / \tau}}{K\left(1-e^{-T_{s} / \tau}\right)} .
$$

Therefore, (17), (20) and (14) yield

$$
C\left(z, T_{S}\right)=\frac{0.4012\left(z-e^{-T_{S} / \tau}\right)}{K\left(1-e^{-T_{S} / \tau}\right)(z-0.9542)},
$$

which is a time-varying model that depends on the sampling period $T_{S}$ and this, in turn, depends on the disturbance period variation $T_{p}$.

The internal stability of the function $P(z)$ introduced in (15) is proved in [16] for any compact interval $\mathscr{T} \subseteq \mathbb{R}+$.

In order to derive the control action, $u_{k}$, applied to the compensator-plant subsystem, it has to be taken into account that the compensator makes the plant time-invariant. Therefore, $u_{k}$ coincides with (18). Finally, the obtention of $\bar{u}_{k}$ according to Figure 4 yields:

$$
\bar{u}_{k}=\frac{0.4012}{K\left(T_{S}\right)} u_{k}-0.4012 \frac{1-K\left(T_{s}\right)}{K\left(T_{S}\right)} u_{k-1}+0.9542 \bar{u}_{k-1}
$$

with $K\left(T_{S}\right)=1-e^{-T_{S} / \tau}$. It can be easily checked than when the sampling interval remains constant at nominal the sampling time, i.e. $T_{s}=\bar{T}$, then $\bar{u}_{k}=u_{k}$.

\section{E. Experimental results}

1) Adaptation of the sampling period: Figure 7 contains experimental results of the repetitive controller designed in Subsection V-B. During the time interval $[0,10] \mathrm{s}$, the reference is maintained constant at the nominal value of $\bar{\omega}=8 \mathrm{rev} / \mathrm{s}:$ it is important to realize that disturbances are almost rejected. At $t=10 \mathrm{~s}$ a ramp reference change, from $\omega=8 \mathrm{rev} / \mathrm{s}$ to $\omega=6.25 \mathrm{rev} / \mathrm{s}$, is introduced in the system; then, the speed is kept constant for $5 \mathrm{~s}$ and finally at $t=20 \mathrm{~s}$ the speed is gradually augmented at a constant acceleration until it reaches the value $\omega=12.5 \mathrm{rev} / \mathrm{s}$ at $t=35 \mathrm{~s}$ : Figure 7 reveals that after $t=10$ s the system can no longer reject 

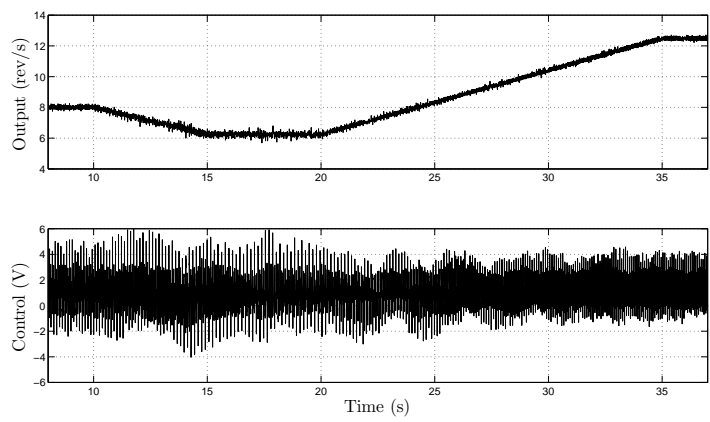

Fig. 8. Closed-loop system behavior using a repetitive controller with adaptive sampling rate.

the disturbances. In addition, the action generated by the control law is also portrayed in Figure 7: it can be seen that the controller generates the necessary action to compensate disturbances when working at the nominal speed, i.e. up to $t=10 \mathrm{~s}$, while when it works at different speeds, i.e. for $t>10 \mathrm{~s}$, the control action is reduced or is not suitable, so disturbances cannot be properly compensated.

Figure 8 shows the results of the same experiment using an adaptive sampling rate, which is accommodated to the desired reference. One may observe that, after a short transient, the controller is capable of preserving the system performance. At the bottom of Figure 8 the control action generated by the control law shows now a proper amplitude for the whole time range. Hence, the controller is capable of rejecting the disturbances, as it was theoretically assured in Subsection V-C.

2) Adaptation-compensation of the sampling period: The system with the adaptation-compensation scheme specified in Subsection V-D is subjected to a speed reference profile identical to the one described in the preceding item. The experimental results are shown in Figure 9. Again, the system performance is preserved. The control action generated by the control law is shown at the bottom of Figure 9.

\section{CONCLUSIONS}

This article introduces two design schemes for digital repetitive control systems subjected to references/disturbances with time-varying period $T_{p}$. Both approaches propose a real-time adaptation of the sampling time of the system, $T_{s}$, in order to maintain the ratio $N=T_{p} / T_{s}$ at a constant level, and one of the structures includes an additional compensator that annihilates the effect of the time-varying sampling in the closed-loop system and forces its behavior to coincide with the one corresponding to an a priori selected nominal sampling period. The stability issue is studied either by means of an LMI gridding method and also using robust control techniques. The LMI gridding approach, besides providing not sufficient but necessary stability conditions, becomes unsuitable for high order systems and/or large analysis intervals due to computational problems, while the robust control approach provides better quality results.
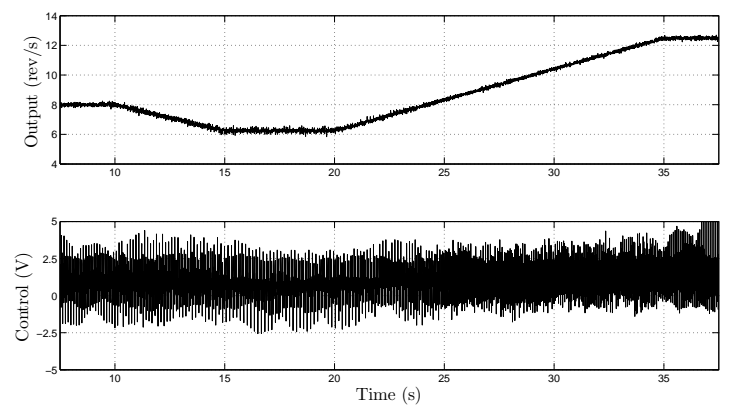

Fig. 9. Closed-loop system behavior using a repetitive controller with adaptation-compensation of the sampling rate.

The adaptation-compensation scheme introduced in Section IV represents an improvement of the preceding setting. However, as an inversion is carried out, it is advisable to deal with minimum phase plant.

\section{REFERENCES}

[1] G. Hillerström and K. Walgama, "Repetitive control theory and applications - A survey," in Proceedings of the 13th IFAC World Congress, Vol. D, 1996, pp. 1-6.

[2] J. Hu, "Variable structure digital repetitive controller," in Proceedings of the 1992 American Control Conference, vol. 2, pp. 2686-2690.

[3] T.-C. Tsao, Y.-X. Qian, and M. Nemani, "Repetitive control for asymptotic tracking of periodic signals with an unknown period," $J$. Dynam. Syst., Meas. Cont., vol. 122, no. 2, pp. 364-369, 2000.

[4] M. Steinbuch, "Repetitive control for systems with uncertain periodtime," Automatica, vol. 38, no. 12, pp. 2103-2109, 2002.

[5] Z. Cao and G. F. Ledwich, "Adaptive repetitive control to track variable periodic signals with fixed sampling rate," IEEE/ASME Trans. Mechatronics, vol. 7, no. 3, pp. 378-384, 2002.

[6] G. Hillerström, "On repetitive control," Ph.D. dissertation, Lulea University of Technology, November 1994.

[7] R. Costa-Castelló, J. Nebot, and R. Griñó, "Demonstration of the internal model principle by digital repetitive control of an educational laboratory plant," IEEE Trans. Educ., vol. 48, no. 1, pp. 73-80, 2005.

[8] K. K. Chew and M. Tomizuka, "Steady-state and stochastic performance of a modified discrete-time prototype repetitive controller," Journal of Dynamic Systems, Measurement, and Control, vol. 112, pp. 35-41, March 1990.

[9] T. Inoue, M. Nakano, T. Kubo, S. Matsumoto, and H. Baba, "High accuracy control of a proton synchroton magnet power supply," in Proceedings of the 8th IFAC World Congress, 1981, pp. 216-220.

[10] W. Rugh, Linear system theory, 2nd Ed. Prentice-Hall, Inc., Upper Saddle River, NJ, 1996.

[11] P. Apkarian and R. Adams, "Advanced gain-scheduling techniques for uncertain systems," IEEE Trans. Control Syst. Tech., vol. 6, no. 1, pp. 21-32, 1998.

[12] A. Sala, "Computer control under time-varying sampling period: An LMI gridding approach," Automatica, vol. 41, no. 12, pp. 2077-2082, 2005 .

[13] G. Ramos, J. Olm, and R. Costa-Castelló, "Digital repetitive control under time-varying sampling period: An lmi stability analysis," in Proceedings of the 18th IEEE International Conference on Control Applications, 2009.

[14] H. Fujioka, "Stability analysis for a class of networked-embedded control systems: A discrete-time approach," in Proceedings of the 2008 American Control Conference, pp. 4997-5002.

[15] Y. Suh, "Stability and stabilization of nonuniform sampling systems," Automatica, vol. 44, no. 12, pp. 3222-3226, 2008.

[16] G. Ramos, J. Olm, and R. Costa-Castelló, "Adaptive compensation strategy for the tracking/rejection of signals with time-varying frequency in digital repetitive control systems," in Proceedings of the 14th IEEE International Conference on Emerging Techonologies and Factory Automation, 2009. 